\title{
Genetic risk score based on fat mass and obesity-associated, transmembrane protein 18 and fibronectin type III domain containing 5 polymorphisms is associated with anthropometric characteristics in South Brazilian children and adolescents
}

\author{
Pâmela F. Todendi ${ }^{1}$, Elisa I. Klinger ${ }^{2}$, Ana C. R. Geraldo ${ }^{3}$, Lucas Brixner ${ }^{3}$, Cézane P. Reuter ${ }^{2}$, \\ Juliana Dal Ri Lindenau ${ }^{4}$, Andréia R. M. Valim ${ }^{2}$ and Marilu Fiegenbaum ${ }^{1,5 *}$ \\ ${ }^{1}$ Graduate Program in Pathology, Federal University of Health Sciences of Porto Alegre (UFCSPA), CEP 90050-170, Porto \\ Alegre/RS, Brazil \\ ${ }^{2}$ Graduate Program in Health Promotion, University of Santa Cruz do Sul (UNISC), CEP 96815-900, Santa Cruz do Sul/RS, \\ Brazil \\ ${ }^{3}$ Biotechnology and Genetics Laboratory, University of Santa Cruz do Sul (UNISC), CEP 96815-900, Santa Cruz do Sul/RS, \\ Brazil \\ ${ }^{4}$ Department of Cellular Biology, Embryology and Genetics, Federal University of Santa Catarina (UFSC), CEP 88040-900, \\ Florianópolis/SC, Brazil \\ ${ }^{5}$ Department of Basic Health Sciences, Federal University of Health Sciences of Porto Alegre (UFCSPA), Porto Alegre/RS, Brazil
}

(Submitted 27 November 2017 - Final revision received 15 August 2018 - Accepted 28 August 2018 - First published online 12 October 2018)

\section{Abstract}

The prevalence of childhood obesity has increased worldwide. Although it is considered a polygenic inheritance disease, little is known about its susceptibility when the additive effect is considered. The aim of this study is to investigate whether the genetic risk score (GRS) based on previously associated obesity polymorphisms (SNP) rs9939609 (fat mass and obesity-associated (FTO)), rs6548238 (transmembrane protein 18 (TMEM18)) and rs16835198 (fibronectin type III domain containing 5 (FNDC5)) could serve as a predictor for anthropometric characteristics in a sample of Brazilian children and adolescents. This is a cross-sectional study with 1471 children and adolescents aged 6-17 years. BMI, waist circumference (WC) and percentage of body fat and metabolic parameters were verified. In all, three SNP were genotyped by TaqMan ${ }^{\mathrm{TM}}$ allelic discrimination. The metabolic and anthropometric parameters were compared between the genotypes, and the unweighted and weighted GRS (GRS and wGRS, respectively) were created to test the additive effect of these genetic polymorphisms on anthropometric parameters. The prevalence of overweight plus obesity was $41 \%$. Significant associations were identified for FTO rs9939609, TMEM18 rs6548238 and FNDC5 rs16835198 and for GRS and wGRS with anthropometric phenotypes. The higher score of wGRS was associated with obesity (OR: $2 \cdot 65,95 \%$ CI 1.40, 5.04, $P=0 \cdot 003$ ) and with greater WC (OR: $2 \cdot 91,95 \%$ CI $1.57,5 \cdot 40, P=0.001$ ). Our results suggest that these genetic variants contribute to obesity susceptibility in children and adolescents and reinforce the idea that the additive effect may be useful to elucidate the genetic component of obesity.

Key words: Obesity: Children: Adolescents: Genetic variants

The prevalence of childhood obesity has increased substantially worldwide and has become a public health problem, particularly over the past two decades ${ }^{(1)}$. Alarming levels of obesity have been observed among children, with the prevalence of overweight and obesity varying significantly between different ethnic groups, sexes and socio-economic categories ${ }^{(1)}$. This complex phenotype results from the interaction of multiple genetic and environmental factors that influence BMI, with an estimated heritability ranging from 40 to $70 \%{ }^{(2)}$.
Common obesity has polygenic inheritance, and genomewide association studies (GWAS) and gene candidate studies have detected several associations between SNP and common diseases, including obesity ${ }^{(3)}$. These association studies identify genetic risk for obesity, which influence the development of obesity and accelerate the weight gain in infancy ${ }^{(3)}$. GWAS in populations of European descent identified more than ninetyseven genetic loci associated with obesity that belong to different pathways, such as insulin secretion, adipogenesis, energy

Abbreviations: BF\%, body fat percentage; FNDC5, fibronectin type III domain containing 5; FTO, fat mass and obesity-associated; GRS, genetic risk score; TMEM18, transmembrane protein 18; WC, waist circumference; wGRS, weighted genetic risk score.

* Corresponding author: Dr M. Fiegenbaum, email mariluf@ufcspa.edu.br 
metabolism and central nervous system $(\mathrm{CNS})^{(4)}$. Using this approach, two new candidate genes related to obesity were identified: transmembrane protein 18 (TMEM18) and the fat mass and obesity-associated (FTO) gene, suggesting that they are responsible for the regulation of body weight, in part, through their actions in the CNS and adipose tissue ${ }^{(5)}$. Through a gene candidate approach, another gene was recently discovered: fibronectin type III domain containing $5(\text { FNDC } 5)^{(6)}$ responsible for encoding the irisin protein, which can modulate lipid metabolism in adipose tissue, suggesting a possible role in energy homoeostasis ${ }^{(7)}$.

Despite great improvements in the discovery of new obesityrelated genes, little is known about susceptibility when the additive effects of these genetic variants are considered, which are important components of the genetic architecture of obesity. This study examined three genes that play a role in the regulation of energy homoeostasis. FTO and TMEM18 genes were replicated in previous studies with children and adolescents ${ }^{(3,8)}$, and FNDC5 may provide new insights into biological factors that contribute to the development of obesity ${ }^{(7)}$. Therefore, the study aims to investigate whether the genetic risk score (GRS) based on previously associated obesity SNP rs9939609 (FTO), rs6548238 (TMEM18) and rs16835198 (FNDC5) could serve as a predictor for anthropometric characteristics in a sample of Brazilian children and adolescents.

\section{Methods}

Sample

This cross-sectional study comprised 1471 children and adolescents aged $6-17$ years, $56 \%$ of whom were female. The students were invited to participate in the study through informed consent signed by parents or guardians. The study protocol was approved by the Research Ethics Committee of the University of Santa Cruz do Sul (UNISC) under number 714 216/14 and the Federal University of Health Sciences of Porto Alegre under number 995 205/15. The participants were consecutively recruited between March 2014 and December 2015 among students from the public and municipal network of Santa Cruz do Sul, Rio Grande do Sul, Brazil. The sample was selected by convenience and was included in the study of healthy subjects, who did not have restrictions for blood collections. The population from Southern Brazil is ethnic mixed ${ }^{(9)}$; thus, the ethnicity determination was made according to Parra et al. ${ }^{(10)}$, based on an evaluation of the following phenotypic characteristics: skin colour in the medial part of the arm; colour and texture of hair; and the shape of the nose and lips. In this sense, $75 \%$ of the subjects were determined as European descendants.

\section{Anthropometric measures}

Body weight and height (coupled stadiometer) were measured using a Welmy balance (Welmy: 15416). BMI was defined by the following formula: BMI = weight $/$ height $^{2}\left(\mathrm{~kg} / \mathrm{m}^{2}\right)$. BMI $Z$-score was classified by World Health Organization ${ }^{(11)}$ according to sex and age, considering low/normal weight $(<1 \mathrm{sD})$, overweight $(>1 \mathrm{sD})$ and obesity ( $>2 \mathrm{sD}$ ). Waist circumference (WC) was determined using inelastic tape, with reference to the narrowest part of the trunk between the ribs and the iliac crest and hip at the level of the greater trochanter. A normal WC was classified as percentile $\leq 80$ and obesity as percentile $>80$, according to previously established criteria $^{(12)}$. The Lange ${ }^{\circledR}$ compass (Beta Technology Incorporated) was used to measure body fat percentage (BF\%), using measurements of triceps and subscapular skinfolds. Subsequently, the equation of slaughter was applied and the data were classified according to the criteria established by Lonman's date, as mentioned by Heyward \& Stolarczyk ${ }^{(13)}$, into two BF\% categories: (1) very low, low and excellent and (2) moderately high, high and very high.

\section{Biochemical analyses}

Blood samples were collected by venepuncture after a 12-h fast. Total cholesterol (TC), HDL-cholesterol, TAG and glucose were measured using Kovalent commercial kits (BioSys Ltda) with a Miura One equipment (ISE). LDL-cholesterol was calculated using the Friedewald equation ${ }^{(14)}$.

\section{Genotyping}

EDTA-anticoagulated whole blood was used for DNA extraction by the salting out method ${ }^{(15)}$. DNA was then quantified using a NanoDrop 2000c spectrophotometer unit (Thermo Scientific). The genotyping of polymorphisms TMEM18 rs6548238, FTO rs9939609 and FNDC5 rs16835198 was performed using Taqman $^{\mathrm{TM}}$ allelic discrimination assays (Applied Biosystems) in StepOne Plus ${ }^{\circledR}$ equipment, according to the manufacturer's instructions. TaqMan ${ }^{\mathrm{TM}}$ assays C_29311887_10 (rs6548238), C_30090620_10 (rs9939609) and C_34204885_10 (rs16835198) and Master Mix PCR Universal were purchased from Applied Biosystems.

\section{Statistical analysis}

The Kolmogorov-Smirnov test was used to verify the normality of the observed variables. Values expressed as the mean and standard deviation were used to describe continuous variables. Allele frequencies were estimated by gene counting. The allelic and genotype distribution between groups and Hardy-Weinberg equilibrium were tested using the $\chi^{2}$ test. A risk score approach was used to evaluate the combined effects of SNP on anthropometric characteristics. The unweighted GRS was constructed using a genotypic score based on the number of unfavourable alleles (those associated with higher levels of BMI $Z$-score and WC) that were carried by each subject for each of the three $\mathrm{SNP}^{(16)}$. Risk alleles were those previously associated with obesity or obesity-related phenotypes in the literature and according to the data of the present study. For SNP rs6548238, rs9939609 and rs16835198, the C, A and T alleles were considered risk alleles, respectively. Weighted GRS (wGRS) was generated by weighting each allele using the natural log of the published OR reported in UK Biobank GWAS results (https://sites.google.com/broadinstitute.org/ukbbgwasresults/). Multiple linear regression analyses were used to adjust 
anthropometrics variables for age, sex and ethnicity. Mean adjusted variables were compared among genotypes and GRS (GRS and wGRS) by ANOVA. Multiple logistic regression analysis was carried out to estimate the OR with $95 \% \mathrm{CI}$ in order to assess genetic risk factors (genotypes, GRS and wGRS) for obesity parameters, as well as to control for confounding factors. A $P$-value $<0.05$ was considered statistically significant. Statistical analyses were performed using SPSS 23.0 for Windows.

\section{Justification of sample size}

The number of students needed to have a representative sample of the city was calculated using the total number of students enrolled using data provided by the sixth Regional Educational Coordinator of Rio Grande do Sul and the Municipal Department of Education of Santa Cruz do Sul. For this purpose, size was calculated using the Nea Research Division ${ }^{(17)}$ formula, suggesting that for 20450 students enrolled the sample should consist of at least 392 students with a $5 \%$ error. The analysed sample (1471 subjects) has a statistical power above $90 \%$ to detect an OR of 1.5 , with a significance level of $5 \%$ with $95 \%$ for SNP with a minor allele frequency of at least $15 \%$.

\section{Results}

Descriptive characteristics of the subjects of this study are presented in Table 1, which comprises $76.5 \%$ of adolescents and $56 \cdot 2 \%$ of female subjects, with a mean age of 11.9 (SD $2 \cdot 8$ ) years. According to the anthropometric characteristics, $23.0 \%$ were overweight, $18.0 \%$ were obese, $21.7 \%$ had high WC and $35.4 \%$ had high $\mathrm{BF} \%$ moderately, high and very high.

The genotypic proportions were in Hardy-Weinberg equilibrium for the three polymorphisms. Frequencies of the minor alleles were $37 \%$ for rs9939609/A (FTO), 14\% for rs6548238/T (TMEM18) and 35\% for rs16835198/T (FNDC5). A comparison of the BMI $Z$-score, $\mathrm{WC}, \mathrm{BF} \%$ and metabolic parameters among genotypes is shown in Table 2. FTO rs9939609 was associated with higher BMI $Z$-score $(P=0.005)$, WC $(P=0.035)$ and TAG $(P=0.007)$, whereas TMEM18 rs6548238 was associated with BMI $Z$-score $(P=0.008)$, WC $(P=0.009)$ and $\mathrm{BF} \%$ $(P=0.024)$. For the rs16835198 at FNDC5 gene, homozygotes TT presented a higher BMI $Z$-score $(P=0.005)$ and WC $(P=0.009)$.

Table 3 shows the means of BMI $Z$-score, WC and BF\% in relation to GRS groups. The means of BMI $Z$-score and WC were found to be higher as the number of risk alleles increased ( $P=0.015, P<0 \cdot 001$, respectively). Although a greater tendency of the means of $\mathrm{BF} \%$ in relation to the GRS groups was observed, this association was not significant.

Fig. 1(a) and (b) show the associations of WC categories (normal percentile $\leq 80$ and high percentile $>80$ ) and BMI $Z$-score (low weight/normal: $<1 \mathrm{sD}$; overweight: $>1$ sD; obesity: $>2 \mathrm{SD}$ ) with GRS. We observed that as the number of risk alleles increased, the percentage of individuals with altered WC $(P=0.001)$ and with obesity $(P=0.017)$ also increased. Among individuals with no risk alleles, none presented altered WC, whereas among those with six risk alleles, $44 \%$ presented WC percentile $>80$ (Fig. 1(a)). Regarding BMI Zscore, $17 \%$ of individuals without risk alleles presented BMI $Z$-score in the overweight range and none was obese. For those with six risk alleles, $24 \%$ were overweight and $40 \%$ were obese (Fig. 1(b)). Association effects of the GRS and wGRS were tested for obesity (BMI $Z$-score $>2$ sD) and altered WC (percentile $>80$ ). The higher score of wGRS was associated with obesity (OR: $2 \cdot 65,95 \%$ CI 1.40, 5.04, $P=0.003$ ) and with greater WC (OR: $2 \cdot 91,95 \%$ CI $1 \cdot 57,5 \cdot 40, P=0 \cdot 001$ ) (Table 4).

Table 1. Descriptive characteristics of the subjects ( $n$ 1471)

(Mean values and standard deviations; numbers and percentages)

\begin{tabular}{|c|c|c|c|c|}
\hline Variables & $n$ & $\%$ & Mean & SD \\
\hline \multicolumn{5}{|l|}{ Sex } \\
\hline Female & 826 & $56 \cdot 2$ & & \\
\hline Male & 645 & 43.8 & & \\
\hline Age (years) & & & 11.9 & $2 \cdot 8$ \\
\hline \multicolumn{5}{|l|}{ Age range* } \\
\hline Children & 345 & 23.5 & & \\
\hline Adolescents & 1126 & $76 \cdot 5$ & & \\
\hline BMI Z-score† & & & 0.78 & 1.3 \\
\hline Low/normal weight & 867 & 58.9 & & \\
\hline Overweight & 339 & 23.0 & & \\
\hline Obesity & 265 & $18 \cdot 0$ & & \\
\hline WC $\ddagger$ & & & 67.4 & $10 \cdot 7$ \\
\hline Normal & 1152 & $78 \cdot 3$ & & \\
\hline High & 319 & 21.7 & & \\
\hline $\mathrm{BF} \%$ & & & 20.8 & $7 \cdot 6$ \\
\hline Very low, low and excellent & 950 & $64 \cdot 6$ & & \\
\hline Moderately high, high and very high & 520 & 35.4 & & \\
\hline
\end{tabular}


Table 2. Association between each SNP of fat mass and obesity-associated (FTO), transmembrane protein 18 (TMEM18) and fibronectin type III domain containing 5 (FNDC5) genes with anthropometric characteristics and metabolic parameters*

(Mean values and standard deviations)

\begin{tabular}{|c|c|c|c|c|c|c|c|c|c|c|c|c|c|c|c|c|c|}
\hline \multirow[b]{2}{*}{ Genes } & \multirow[b]{2}{*}{$n$} & \multicolumn{2}{|c|}{ BMI Z-score } & \multicolumn{2}{|c|}{ WC } & \multicolumn{2}{|c|}{$\mathrm{BF} \%$} & \multicolumn{2}{|c|}{$\mathrm{TC}(\mathrm{mmol} / \mathrm{l})$} & \multicolumn{2}{|c|}{ LDL-C (mmol/l) } & \multicolumn{2}{|c|}{$\mathrm{HDL}-\mathrm{C}(\mathrm{mmol} / \mathrm{l})$} & \multicolumn{2}{|c|}{ TAG $(\mathrm{mmol} / \mathrm{l})$} & \multicolumn{2}{|c|}{ Glucose $(\mathrm{mmol} / \mathrm{l})$} \\
\hline & & Mean & SD & Mean & SD & Mean & SD & Mean & $\mathrm{SD}$ & Mean & SD & Mean & SD & Mean & SD & Mean & SD \\
\hline \multicolumn{18}{|c|}{ FTO rs9939609 } \\
\hline TT & 583 & 0.70 & $1 \cdot 26$ & $66 \cdot 7$ & 9.8 & 20.7 & $7 \cdot 3$ & $4 \cdot 10$ & 0.86 & $2 \cdot 11$ & 0.73 & 1.62 & 0.30 & 0.79 & 0.37 & 4.93 & 0.60 \\
\hline $\mathrm{TA}$ & 689 & 0.80 & $1 \cdot 28$ & $67 \cdot 5$ & $10 \cdot 7$ & 20.7 & $7 \cdot 7$ & $4 \cdot 11$ & 0.82 & $2 \cdot 12$ & 0.70 & 1.60 & 0.30 & 0.85 & 0.42 & 5.05 & 1.90 \\
\hline $\mathrm{AA}$ & 198 & 0.96 & $1 \cdot 33$ & $69 \cdot 1$ & $12 \cdot 8$ & $21 \cdot 3$ & $8 \cdot 1$ & 4.05 & 0.90 & 2.07 & 0.74 & 1.58 & 0.31 & 0.86 & 0.50 & 4.91 & 0.60 \\
\hline$P$ & & \multicolumn{2}{|c|}{0.005} & \multicolumn{2}{|c|}{0.035} & \multicolumn{2}{|c|}{0.380} & \multicolumn{2}{|c|}{0.755} & \multicolumn{2}{|c|}{0.748} & \multicolumn{2}{|c|}{0.139} & \multicolumn{2}{|c|}{0.007} & \multicolumn{2}{|c|}{0.208} \\
\hline \multicolumn{18}{|c|}{ TMEM18 rs6548238 } \\
\hline $\mathrm{CC}$ & 1083 & 0.85 & $1 \cdot 28$ & $67 \cdot 9$ & $10 \cdot 9$ & $21 \cdot 1$ & $7 \cdot 7$ & $4 \cdot 11$ & 0.85 & $2 \cdot 12$ & $0 \cdot 71$ & 1.61 & 0.31 & 0.84 & 0.43 & $5 \cdot 00$ & 1.52 \\
\hline CT & 355 & 0.60 & $1 \cdot 28$ & $66 \cdot 4$ & $10 \cdot 0$ & $20 \cdot 1$ & $7 \cdot 2$ & 4.07 & 0.84 & 2.09 & 0.75 & 1.61 & 0.27 & 0.80 & 0.36 & 5.00 & 0.86 \\
\hline TT & 33 & 0.50 & 0.99 & $64 \cdot 0$ & $8 \cdot 7$ & $19 \cdot 5$ & $6 \cdot 4$ & 3.97 & 0.95 & $2 \cdot 11$ & 0.77 & 1.52 & 0.25 & 0.74 & 0.39 & $4 \cdot 85$ & 0.50 \\
\hline$P$ & & \multirow{2}{*}{\multicolumn{2}{|c|}{0.008}} & \multicolumn{2}{|c|}{0.009} & \multicolumn{2}{|c|}{0.024} & \multicolumn{2}{|c|}{0.441} & \multicolumn{2}{|c|}{$0 \cdot 760$} & \multicolumn{2}{|c|}{0.290} & \multicolumn{2}{|c|}{0.069} & \multicolumn{2}{|c|}{0.855} \\
\hline \multicolumn{16}{|c|}{ FNDC5 rs16835198 } & & \\
\hline $\mathrm{GG}$ & 624 & 0.82 & 1.25 & $67 \cdot 8$ & $10 \cdot 5$ & $20 \cdot 9$ & $7 \cdot 6$ & $4 \cdot 10$ & 0.87 & $2 \cdot 14$ & 0.73 & 1.60 & 0.30 & 0.82 & 0.44 & $5 \cdot 00$ & 1.94 \\
\hline GT & 658 & 0.71 & 1.26 & $66 \cdot 5$ & 9.9 & $20 \cdot 3$ & $7 \cdot 2$ & $4 \cdot 12$ & 0.83 & $2 \cdot 10$ & 0.71 & 1.63 & 0.30 & 0.83 & 0.40 & $5 \cdot 00$ & 0.72 \\
\hline TT & 189 & 0.91 & 1.42 & 68.9 & 13.4 & $22 \cdot 0$ & $8 \cdot 6$ & 4.03 & 0.81 & 2.06 & $0 \cdot 70$ & 1.60 & 0.31 & 0.80 & 0.40 & 4.92 & 0.60 \\
\hline$P$ & & \multicolumn{2}{|c|}{0.005} & \multicolumn{2}{|c|}{0.009} & \multicolumn{2}{|c|}{$0 \cdot 100$} & 0.2 & & & & & & 0.2 & & & \\
\hline
\end{tabular}

WC, waist circumference (percentile); BF\%, body fat percentage; TC, total cholesterol.

${ }^{*}$ ANOVA, adjusted by sex, age and ethnicity.

Table 3. Anthropometric characteristics according unweighted and weighted genetic risk score of the three SNP in fat mass and obesityassociated (FTO), transmembrane protein 18 (TMEM18) and fibronectin type III domain containing 5 (FNDC5) genes with anthropometric characteristics and metabolic parameters

(Mean values and standard deviations)

\begin{tabular}{|c|c|c|c|c|c|c|c|}
\hline & \multirow[b]{2}{*}{$n$} & \multicolumn{2}{|c|}{ BMI Z-score } & \multicolumn{2}{|c|}{ WC (cm) } & \multicolumn{2}{|c|}{$\mathrm{BF} \%$} \\
\hline & & Mean & SD & Mean & SD & Mean & SD \\
\hline \multicolumn{8}{|l|}{ GRS† } \\
\hline No risk allele & 6 & 0.30 & 0.83 & 59.9 & 5.6 & $19 \cdot 6$ & $6 \cdot 0$ \\
\hline 01 & 83 & 0.76 & 1.15 & $68 \cdot 0$ & $10 \cdot 4$ & $21 \cdot 6$ & $7 \cdot 6$ \\
\hline 02 & 309 & 0.65 & 1.29 & $66 \cdot 4$ & $10 \cdot 0$ & $20 \cdot 0$ & $7 \cdot 2$ \\
\hline 03 & 521 & 0.77 & $1 \cdot 22$ & $67 \cdot 3$ & 9.8 & $20 \cdot 7$ & $7 \cdot 2$ \\
\hline 04 & 405 & 0.80 & 1.33 & 67.9 & $10 \cdot 6$ & $20 \cdot 9$ & 7.9 \\
\hline 05 & 122 & 1.01 & 1.37 & $69 \cdot 6$ & $13 \cdot 0$ & 21.9 & 8.6 \\
\hline 06 & 25 & 1.37 & 1.34 & $75 \cdot 3$ & $19 \cdot 4$ & $23 \cdot 8$ & 8.3 \\
\hline$P$ & & \multicolumn{2}{|c|}{0.015} & \multicolumn{2}{|c|}{$<0.001$} & \multicolumn{2}{|c|}{0.177} \\
\hline \multicolumn{8}{|l|}{ wGRS } \\
\hline 0 & 6 & 0.30 & 0.84 & $59 \cdot 9$ & $5 \cdot 6$ & $19 \cdot 6$ & $6 \cdot 0$ \\
\hline w01 (0.01-0.09)‡ & 174 & 0.60 & 1.21 & $66 \cdot 8$ & 9.4 & 20.5 & $7 \cdot 0$ \\
\hline w02 (0.10-0.14)‡ & 586 & 0.71 & 1.29 & $66 \cdot 4$ & $10 \cdot 1$ & $20 \cdot 5$ & $7 \cdot 3$ \\
\hline w03 $(0.15-0.19) \ddagger$ & 526 & 0.86 & 1.26 & $68 \cdot 1$ & $10 \cdot 7$ & $21 \cdot 1$ & 7.8 \\
\hline w04 $(0.20-0.24) \ddagger$ & 96 & 0.93 & 1.35 & $68 \cdot 0$ & 11.4 & $21 \cdot 3$ & 8.3 \\
\hline w05 (0.25-0.27)‡ & 83 & 1.04 & 1.36 & 71.0 & 14.9 & $21 \cdot 7$ & 8.1 \\
\hline$P$ & & \multicolumn{2}{|c|}{0.021} & \multicolumn{2}{|c|}{0.001} & \multicolumn{2}{|c|}{0.506} \\
\hline
\end{tabular}

WC, waist circumference; BF\%, body fat percentage; GRS, genetic risk score; wGRS, weight genetic risk score.

* Association analysis adjusted by sex, age and ethnicity.

† GRS: no risk allele, 01 - one risk allele, 02 - two risk alleles, 03 - three risk alleles, 04 - four risk alleles, 05 - five risk alleles and 06 - six risk alleles.

$\ddagger$ Weighted value: number of alleles risk multiplied by the natural log of the published OR.

\section{Discussion}

The objective of this study was to investigate whether there is an additive effect of SNP rs9939609 (FTO), rs6548238 (TMEM18) and rs16835198 (FNDC5) on anthropometric characteristics in Brazilian children and adolescents. The comparison between the anthropometric variables evaluated among
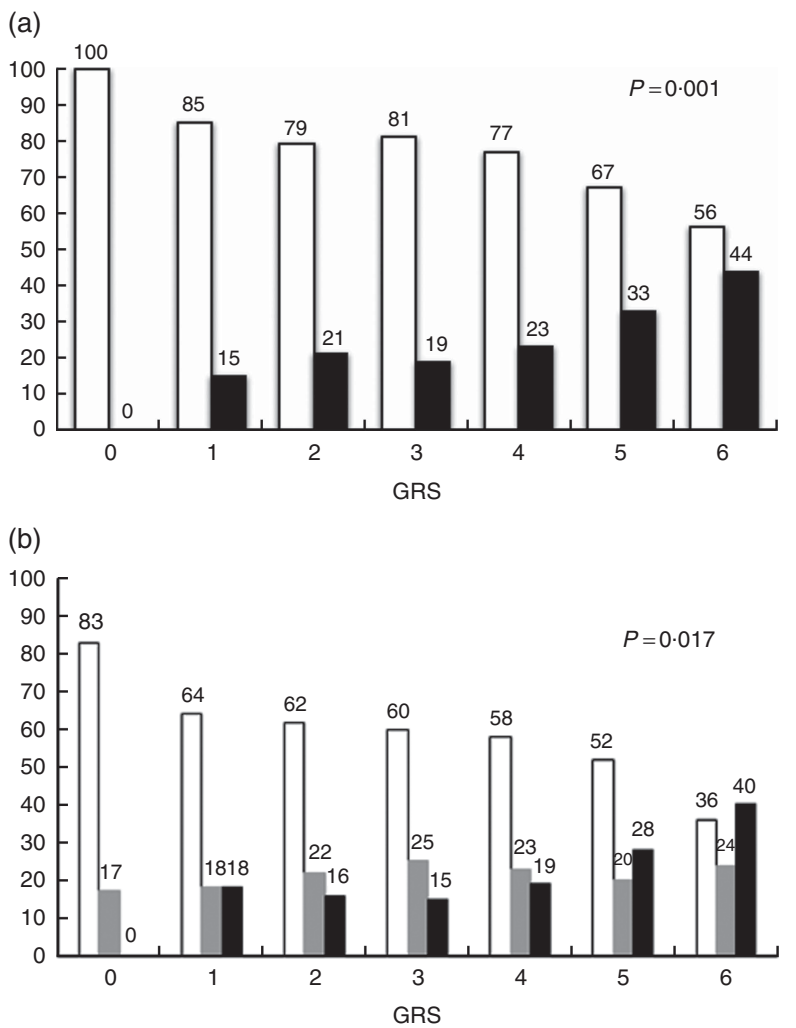

Fig. 1. (a) Association of categories of waist circumference (WC) with genetic risk score (GRS). $\square$, WC percentile <80; $\square$, WC percentile $\geq 80$. (b) Association of BMI Z-score categories with GRS. Z-score: low/normal weight, <1sD ( $\square$ ); overweight, >1sD ( $\square$ ); obesity, >2sD ( $\square$ ). GRS: 0, no risk allele; 1 , one risk allele; 2 , two risk alleles; 3 , three risk alleles; 4 , four risk alleles; 5 , five risk alleles; 6 , six risk alleles.

genotypes of each SNP replicated the significant associations that have already been observed in other studies on obesity, mainly WC and BMI. The main finding of this study is the cumulative risk effect for obesity and altered WC according to 
Table 4. Multiple logistic regression for obesity (BMI Z-score $>2 \mathrm{sD}$ ) and for high waist circumference (WC percentile $>80$ ) according unweighted genetic risk score (GRS) and weighted genetic risk score (wGRS) of the three SNP in fat mass and obesity-associated (FTO), transmembrane protein 18 (TMEM18) and fibronectin type III domain containing 5 (FNDC5) genes* (Odds ratios and $95 \%$ confidence intervals)

\begin{tabular}{|c|c|c|c|c|c|c|}
\hline & \multicolumn{3}{|c|}{ Obesity } & \multicolumn{3}{|c|}{ WC percentile $>80$} \\
\hline & OR & $95 \% \mathrm{Cl}$ & $P$ & OR & $95 \% \mathrm{Cl}$ & $P$ \\
\hline \multicolumn{7}{|l|}{ GRS† } \\
\hline $0-1$ & 1 & & & 1 & & \\
\hline 2 & 0.80 & $0.42,1.52$ & 0.497 & 1.60 & $0.82,3 \cdot 14$ & 0.170 \\
\hline 3 & 0.81 & $0.44,1.50$ & 0.509 & 1.44 & $0.75,2.76$ & 0.275 \\
\hline 4 & 1.03 & $0.55,1.91$ & 0.927 & 1.85 & $0.96,3.56$ & 0.067 \\
\hline 5 & $1 \cdot 71$ & $0.85,3.43$ & 0.131 & 2.97 & $1.44,6 \cdot 13$ & 0.003 \\
\hline 6 & 3.38 & $1 \cdot 25,9 \cdot 17$ & 0.017 & 4.99 & $1 \cdot 82,13 \cdot 70$ & 0.002 \\
\hline \multicolumn{7}{|l|}{ wGRS } \\
\hline w0-1 & 1 & & & 1 & & \\
\hline w02 & $1 \cdot 11$ & $0.68,1.80$ & 0.676 & $1 \cdot 34$ & $0.84,2 \cdot 12$ & 0.218 \\
\hline w03 & $1 \cdot 27$ & $0.78,2.07$ & 0.328 & 1.74 & $1 \cdot 10,2 \cdot 76$ & 0.018 \\
\hline w04 & $2 \cdot 15$ & $1 \cdot 14,4.06$ & 0.018 & 1.92 & $1.03,3.58$ & 0.040 \\
\hline w05 & 2.65 & $1.40,5.04$ & 0.003 & 2.91 & $1.57,5.40$ & 0.001 \\
\hline
\end{tabular}

the GRS created to evaluate the combined effects of the three SNP. The BF\% showed no association with the GRS; supposedly other genes are associated with this adiposity variable.

Our data show that the allelic frequencies found are similar to previous studies ${ }^{(8,18,19)}$. Minor allelic frequencies of rs9939609/A (FTO) and rs6548238/T (TMEM18) were 35 and 15\%, respectively, in other studies with Brazilian population ${ }^{(8,18)}$. The rs16835198/T allele (FNDC5) has not yet been genotyped in Brazilian populations, but a study with European offspring shows a frequency of $35 \%$ for the minor allele ${ }^{(19)}$, confirming the same value found in our study.

Variants of the FTO and TMEM18 genes were initially associated with obesity in adults ${ }^{(20,21)}$; the FNDC5 gene variant appears to influence glycolipid metabolism in overweight/ obese adult subjects $^{(22)}$. In addition, researchers have recently investigated the effect of these variants on the risk of obesity as early as childhood and adolescence ${ }^{(3,8)}$. Early obesity, particularly in children, is highly predictive of obesity in adulthood, especially when one (or both) of the parents is obese ${ }^{(23)}$. This early obesity is one of the determinants of cardiometabolic risk in adulthood ${ }^{(24)}$

The FTO gene is involved in the stimulation of food intake and is highly expressed in the human hypothalamus, pituitary gland and adrenal glands, suggesting a potential role in the hypothalamic-pituitary-adrenal axis and implying the regulation of body weight. Several potentially functional SNP at the FTO locus are highly associated with the early onset of obesity and severe obesity in European populations ${ }^{(25)}$. The most studied SNP of this gene, rs9939609, was evaluated in this study and was consistently associated with higher values of BMI $Z$-score, WC and TAG $(P<0.05)$ in children and adolescents. Other studies performed with this same age group and populations of European and Asian and Amerindian origin also show an association of this SNP rs9939609 with higher means of BMI $Z$-score, WC and TAG $(P<0 \cdot 05)^{(20,26,27)}$.

The TMEM18 gene is expressed or known to act on the CNS, although it also exhibits peripheral functions related to adipose tissue ${ }^{(28)}$. To better understand the relation of this gene to obesity, this study examined the relationship between variant rs6548238 and anthropometric characteristics. We observed that $\mathrm{C}$ allele carriers presented higher BMI $Z$-score, WC and $\mathrm{BF} \%$ values $(P<0.05)$. Other studies involving children and adolescents have also shown an association of this variant with BMI $Z$ score, WC and BF\% in populations of Mexican ${ }^{(29)}$, European $^{(30)}$ and New Zealand origin ${ }^{(31)}$.

Since its discovery in 2012, the irisin protein encoded by the FNDC5 gene has attracted interest as a potential health mediator, promoting effects on physical exercise. It is considered to be a novel hormone-like myokine released by skeletal muscle during exercise to improve obesity and glucose dysfunction, stimulating the darkening of white adipose tissue ${ }^{(6)}$. Evidence indicates that irisin is expressed robustly, not only in skeletal muscle $e^{(6)}$ but also in various regions of brain tissue ${ }^{(32)}$. To the best of our knowledge, there is only one study with adults and a population of Chinese origin that associated the rs16835198 SNP of the FNDC5 gene with glycolipid metabolism in overweight/obese subjects ${ }^{(22)}$. However, this study is the first to observe the relationship of this SNP with higher means of BMI $Z$-score and WC $(P<0.005)$ in children and adolescents.

Although GWAS have identified a significant number of SNP associated with many complex human characteristics, the susceptibility loci identified to date may account for only a small fraction of the genetic risk ${ }^{(33)}$. Although several factors such as rare genetic variants, structural variations, epigenetic modifications and gene-environment interactions may contribute to 'missing heritability'(34), the additive effect of several SNP has been poorly explored and becomes an important component of 
multifactorial genetic diseases owing to the complexity of biological systems ${ }^{(35)}$. In this sense, numerous studies have already evaluated the SNP studied in this research with obesity, but very few studies have evaluated the simultaneous effect of the presence of the risk alleles of each SNP.

Thus, it was observed in this study that the additive effect among the studied SNP was higher as the number of risk alleles of individuals with altered WC $(P<0.001)$ and with obesity increased $(P=0.015)$. In addition, our data showed that subjects with higher GRS are associated with higher risk of obesity and altered WC (Table 4). The association was significant both for unweighted GRS and wGRS. To date, only four studies on additive effects have shown an association of obesity and the rs9939609 SNP of the FTO gene, one study has shown an association with the variant rs6548238 of the TMEM18 gene and no study has uncovered an association with the variant rs16835198 of the FNDC5 gene.

A study with children and adolescents of European origin tested the combination of $A C E$ (rs4646994), FTO (rs9939609), $M C 4 R$ (rs17782313) and PPARG (rs1801282) SNP with obesity, showing influence of $M C 4 R$ and $P P A R G$ with higher $\mathrm{BMI}^{(36)}$. Another study with an adult population of Indian origin evaluated fifty-five SNP in twenty-eight genes. However, the SNP FTO (rs9939609), iroquois homeobox 3 (IRX3) (rs3751723), transcription factor 7 like 2 (TCF $7 L 2$ ) (rs7903146) and TMEM18 (rs6548238) were observed to be the main SNP that contribute to the risk of obesity linked to $\mathrm{BMI}^{(37)}$. A study of Indian adults tested the additive effect of SNP FTO (rs8050136, rs1421085, rs9939609, rs17817449) and IRX3 (rs3751723) and observed the association of these genes with the risk for obesity ${ }^{(38)}$. Finally, a study of Chinese adults tested the additive effect among eleven variants of the FTO gene (among them rs9939609), TSPAN8 and TCF7L2, and they were associated with type 2 diabetes mellitus and obesity $^{(33)}$

Although FTO and TMEM18 SNP were associated with obesity in many studies ${ }^{(4,8,20,30,31)}$, some researchers found no association of the rs6548238 (TMEM18) ${ }^{(39)}$ and rs16835198 $(F N D C 5)^{(40)}$ variants with adiposity parameters. To the best of our knowledge, this is the first study to test the variant rs16835198 of the FNDC5 gene in obese children and adolescents and to analyse its cumulative effect with other SNP. It is also the first study to analyse the cumulative effect of these three SNP in a sample composed of Brazilian children and adolescents. However, there were some limitations. Of all Brazilian regions, European immigrants predominantly populate the southern region. According to Parra et al. ${ }^{(10)}$, in Brazil the colour determined by the physical evaluation is a weak predictor of genomic ancestry. This factor may limit this study, as the ancestry was self-declared and may not reflect the real ancestry of the research subjects. Sample size is representative of this population, although some studies use larger sample size in this type of study. Further, sufficient statistical power was allowed for our analyses, and these data can be incorporated into future meta-analyses.

In conclusion, three SNP analysed of the genes FTO, TMEM18 and FNDC5 were found to be associated with the susceptibility of developing greater BMI $Z$-score and WC in a sample composed of Brazilian children and adolescents. These findings are consistent with other studies that consistently associate these genes with obesity. Together with our results, these data corroborate the idea that the cumulative effect may be useful to understand the genetic component of obesity. The results of this study also suggest that this method may be an option for future studies, mainly in order to find the missing heritability.

\section{Acknowledgements}

The authors thank all volunteers who participated in this study, and the Biotechnology and Genetics Laboratory of UNISC. The authors also thank with great affection the collaborator Miria S. Burgos (in memory).

This research was supported by the Brazilian Agencies Foundation for Research Support of Rio Grande do Sul (FAPERGS), the National Counsel of Technological and Scientific Development (CNPq) and Coordination of Improvement of Higher Level Personnel (CAPES).

P. F. T. was involved in data collection, data analyses, data interpretation and manuscript writing. E. I. K., A. C. R. G. and L. B. contributed to data collection and genotyping. C. P. R. and A. R. M. V. contributed to study design, data interpretation and manuscript writing. J. D. R. L. and M. F. contributed to study design, data analyses, data interpretation and manuscript writing. All authors contributed to the revision of the manuscript and approved the final manuscript.

The authors declare that there are no conflicts of interest.

\section{References}

1. Lobstein T \& McPherson K (2016) Nothing new in UK's strategy on childhood obesity. Lancet 388, 853-854.

2. Zaitlen N, Kraft P, Patterson N, et al. (2013) Using extended genealogy to estimate components of heritability for 23 quantitative and dichotomous traits. PLOS Genet 9, e1003520.

3. Steinsbekk S, Belsky D, Guzey IC, et al. (2016) Polygenic risk, appetite traits, and weight gain in middle childhood: a longitudinal study. JAMA Pediatr 170, e154472.

4. Locke AE, Kahali B, Berndt SI, et al. (2015) Genetic studies of body mass index yield new insights for obesity biology. Nature 518, 197-206.

5. Edward ARN, Stephen AH, Lynn R, et al. (2016) Birth weight modifies the association between central-nervous-system gene variation and adult body mass index. J Hum Genet 61, 193-198.

6. Boström P, Wu J, Jedrychowski MP, et al. (2012) A PGC1alpha-dependent myokine that drives brown-fat-like development of white fat and thermogenesis. Nature 481, 463-468.

7. Ge X, Sathiakumar D, Lua BJG, et al. (2017) Myostatin signals through miR-34a to regulate Fndc 5 expression and browning of white adipocytes. Int J Obes (Lond) 41, 137-148.

8. Zandoná MR, Sangalli CN, Campagnolo PD, et al. (2017) Validation of obesity susceptibility loci identified by gemomewide as association studies in early childhood in South Brazilian children. Pediatr Obes 12, 85-92.

9. Santos SA (2002) Historical roots of the "Whitening" of Brazil. Latin Am Perspect 122, 61-82.

10. Parra FC, Amado RC, Lambertucci JR, et al. (2003) Color and genomic ancestry in Brazilians. Proc Natl Acad Sci U S A 100, 177-182. 
11. WHO (2007) Growth reference data for 5-19 years. http:// www.who.int/growthref/en/ (accessed May 2017).

12. Fernández JR, Redden DT, Pietrobelli A, et al. (2004) Waist circumference percentiles in nationally representative samples of African-American, European-American, and MexicanAmerican children and adolescents. J Pediatr 145, 439-444.

13. Heyward VH \& Stolarczyk LM (2000) Composição corporal aplicada. São Paulo: Manole.

14. Friedewald WT, Levy RI \& Fredrickson DS (1972) Estimation of the concentration of low-density lipoprotein cholesterol in plasma, without use of the preparative ultracentrifuge. Clin Chem 18, 499-502.

15. Miller SA, Dykes DD \& Polesky HF (1988) A simple salting out procedure for extracting DNA from human nucleated cells. Nucleic Acids Res 16, 1215

16. Kathiresan S, Melander O, Anevski D, et al. (2008) Polymorphisms associated with cholesterol and risk of cardiovascular events. $N$ Engl J Med 358, 1240-1249.

17. Christensen LB (1980) Experimental Methodology. Boston, MA: Allyn/Bacon.

18. Reuter CP, Burgos MS, Bernhard JC, et al. (2016) Association between overweight and obesity in schoolchildren with rs9939609 polymorphism (FTO) and family history for obesity. J Pediatr (Rio J) 92, 493-498.

19. Staiger H, Böhm A, Scheler M, et al. (2013) Common genetic variation in the human FNDC5 locus, encoding the novel muscle-derived 'browning' factor irisin, determines insulin sensitivity. PLOS ONE 8, e61903.

20. Frayling TM, Timpson NJ, Weedon MN, et al. (2007) A common variant in the FTO gene is associated with body mass index and predisposes to childhood and adult obesity. Science 316, 889-894.

21. Hotta K, Nakamura M, Nakamura T, et al. (2009) Association between obesity and polymorphisms in SEC16B, TMEM18, GNPDA2, BDNF, FAIM2 and MC4R in Japanese population. J Hum Genet 54, 727-731.

22. Tang S, Zhang R, Jiang F, et al. (2014) An interaction between a $F N D C 5$ variant and obesity modulates glucose metabolism in a Chinese Han population. PLOS ONE 9, e109957.

23. Whitaker RC, Wright JA, Pepe MS, et al. (1997) Predicting obesity in young adulthood from childhood and parental obesity. $N$ Engl J Med 337, 869-873.

24. Estampador AC \& Franks PW (2014) Genetic and epigenetic catalysts in early-life programming of adult cardiometabolic disorders. Diabetes Metab Syndr Obes 7, 575-586.

25. Dina C, Meyre D, Gallina S, et al. (2007) Variation in FTO contributes to childhood obesity and severe adult obesity. Nat Genet 39, 724-726.

26. Liu C, Mou S \& Cai Y (2013) FTO gene variant and risk of overweight and obesity among children and adolescents: a systematic review and meta-analysis. PLOS ONE 8, e82133.
27. Freathy RM, Timpson NJ, Lawlor DA, et al. (2008) Common variation in the FTO gene alters diabetes-related metabolic traits to the extent expected given its effect on BMI. Diabetes 57, 1419-1426.

28. Willer CJ, Speliotes EK, Loos RJ, et al. (2009) Six new loci associated with body mass index highlight a neuronal influence on body weight regulation. Nat Genet 41, 25-34.

29. León-Mimila P, Villamil-Ramírez H, Villalobos-Comparán M, et al. (2013) Contribution of common genetic variants to obesity and obesity-related traits in Mexican children and adults. PLOS ONE 8, e70640.

30. Almén MS, Jacobsson JA, Shaik JH, et al. (2010) The obesity gene, TMEM18, is of ancient origin, found in majority of neuronal cells in all major brain regions and associated with obesity in severely obese children. BMC Med Genet 11, 58.

31. Krishnan M, Thompson JMD, Mitchell EA, et al. (2017) Analysis of association of gene variants with obesity traits in New Zealand European children at 6 years of age. Mol Biosyst 13, 1524-1533.

32. Dun SL, Lyu RM, Chen YH, et al. (2013) Irisin-immunoreactivity in neural and non-neural cells of the rodent. Neuroscience $\mathbf{2 4 0}$, $155-162$.

33. Zhu Z, Tong X, Zhu Z, et al. (2013) Development of GMDRGPU for gene-gene interaction analysis and its application to WTCCC GWAS data for type 2 diabetes. PLOS ONE $\mathbf{8}$, e61943.

34. Eichler EE, Flint J, Gibson G, et al. (2010) Missing heritability and strategies for finding the underlying causes of complex disease. Nat Rev Genet 11, 446-450.

35. Cordell HJ (2009) Detecting gene-gene interactions that underlie human diseases. Nat Rev Genet 10, 392-404.

36. Bordoni L, Marchegiani F, Piangerelli M, et al. (2017) Obesityrelated genetic polymorphisms and adiposity indices in a young Italian population. IUBMB Life 69, 98-105.

37. Srivastava A, Mittal B, Prakash J, et al. (2017) A multianalytical approach to evaluate the association of 55 SNPs in 28 genes with obesity risk in North Indian adults. Am J Hum Biol 29, e22923.

38. Srivastava A, Mittal B, Prakash J, et al. (2016) Association of FTO and IRX3 genetic variants to obesity risk in north India. Ann Hum Biol 43, 451-456.

39. García-Solís P, Reyes-Bastidas M, Flores K, et al. (2016) Fat mass obesity-associated (FTO) (rs9939609) and melanocortin 4 receptor (MC4R) (rs17782313) SNP are positively associated with obesity and blood pressure in Mexican school-aged children. Br J Nutr 116, 1834-1840.

40. Tanisawa K, Taniguchi H, Sun X, et al. (2014) Common single nucleotide polymorphisms in the FNDC5 gene are associated with glucose metabolism but do not affect serum irisin levels in Japanese men with low fitness levels. Metabolim $\mathbf{6 3}$, $574-583$. 